\title{
Global miRNA Expression Analysis Identifies miR- 194, miR-100, miR-125b and miR-199a as Promising Biomarkers for Gastric Cancer and miR-194 Inhibits Gastric Cancer Cell Growth by Targeting CCND1
}

\section{Dandan Li}

Hubei University of Medicine

Jingjie Wang

Hubei University of Medicine

Meixin Zhang

Hubei University of Medicine

Xinhui Hu

Hubei University of Medicine

Jiajun She

Hubei University of Medicine

Mengyu Cai

Hubei University of Medicine

Lijuan Yang

Hubei University of Medicine

Weijia Liu

Hubei University of Medicine

Qiwen Hu

Hubei University of Medicine

Xuemei Qiu

Hubei University of Medicine

Shanshan Qin ( $D$ qinss77@163.com )

Hubei University of Medicine https://orcid.org/0000-0002-8527-5278

Primary research

Keywords: miR-194, prognostic biomarker, CCND1, cell cycle, gastric cancer

Posted Date: August 14th, 2020

DOI: https://doi.org/10.21203/rs.3.rs-57264/v1 
License: (c) (i) This work is licensed under a Creative Commons Attribution 4.0 International License. Read Full License 


\section{Abstract}

\section{Background}

Different gastric cancer (GC) subtypes usually possess distinct clinical outcomes. The function of miR194 in gastric cancer remains unclear and controversial. This study aimed to identify potential microRNAs that differentially expressed in subtypes and to elucidate the molecular mechanisms of miR194 in GC.

\section{Methods}

Comprehensive miRNA expression analysis was performed using the available miRNA-seq data from TCGA stomach cancer cohort. The preferences of miR-194 in regulating target genes were determined by RNA sequencing studies. The function of miR-194 in GC was explored in GC cell lines by performing qRTPCR assays, western blot assays, cell proliferation assays, luciferase report assays and flow cytometry assay.

\section{Results}

In this study, we identified a series of miRNAs that can serve as prognostic biomarkers for GC. Among them, miR-100, miR-125b, miR-199a and miR-194 were the 4 most promising prognostic biomarkers in GC due to their significant associations with various clinical characteristics of patients. MiR-100, miR-125b and miR-199a predicted poor prognosis in GC, while miR-194 predicted favorable prognosis in GC.

Besides, we provided the first comprehensive transcriptome analysis about miR-194 in GC. The results showed that miR-194 tended to regulated target genes by binding on their 3' untranslated regions in a 7mer-A1 or 7-mer-m8 or 8-mer manner. The KEGG pathway analysis showed that cell cycle was one of the most affected pathways by miR-194 in GC. Moreover, CCND1 was proved to be a novel target gene of miR-194 in GC. Additionally, the downregulation of CCND1 by miR-194 in GC further led to cell growth inhibition and cell cycle arrest.

\section{Conclusions}

MiR-100, miR-125b, miR-199a and miR-194 could serve as prognostic and diagnostic biomarkers for GC. MiR-194 might suppress GC cell growth mainly through targeting CCND1 and induction of cell cycle arrest.

\section{Background}

Gastric cancer (GC) is the fourth most diagnosed type of cancer and the third most common cause of cancer-related death worldwide[1]. Approximately 989,600 people worldwide are diagnosed with stomach cancer each year. More than half of those cases are diagnosed in East Asia[2]. However, many patients with gastric cancer are diagnosed with advanced malignant proliferation, extensive invasion and distant 
metastasis. Hence, the 5 years survival rate of patients with advanced gastric cancer was still unsatisfactory. Characteristic progressive tumorigenesis and distant metastasis may contribute to the poor overall prognosis a lot in gastric cancer [3]. Therefore, it is essential to develop accurate biomarkers to predict cancer stage or reflect an individual's cancer risk, which would be very useful for reducing GC mortality.

Mature microRNAs (miRNA) are a group of small non-coding RNAs with 19-24 nucleotides. Mature miRNAs bind to the specific regions in the 3 ' untranslated regions (3'-UTRs) of the target mRNAs and negatively regulate their expression at post-transcription level[4]. Increasing studies have shown that miRNA plays a very important role in tumorigenesis by affecting multiple cellular processes, such as epithelial-mesenchymal transition (EMT), cell invasion, cell apoptosis, cell proliferation, etc.[5]. Besides, miRNA expression patterns can be used to distinguish tumor subtypes and predict clinical outcomes[6]. Therefore, the identification of GC-associated miRNAs as biomarkers for early tumor detection, prognosis and treatment is of great importance.

The function of miR-194 in stomach cancer still remains unclear and controversial. As early as 2012, Song et al. has reported that overexpression of miR-194 predicts better prognosis in GC [7]. After that, several studies have shown that miR-194 inhibited gastric cancer progression via targeting FOXM1[8], RBX1[9] and KDM5B[10]. However, another recent study reported that miR-194 promotes GC cell proliferation and migration by activating Wnt signaling, at least in part, via suppression of SUFU, which suggested miR-194 play oncogenic role in GC[11]. Due to there must be a large number of gene transcripts downregulated by miR-194, those studies about the role of miR-194 in GC were inevitably limited. Therefore, it's necessary to consider preferences of miR-194 in regulating target genes.

In this study, we conducted comprehensive miRNA expression analysis in different GC subtypes using TCGA data, and identified a panel of miRNAs (miR-100, miR-125b, miR-199a and miR-194) that can serve as prognostic and diagnostic biomarkers for GC. In addition, RNA sequencing studies were conducted to determine the most affected pathways and target genes by miR-194 in GC. Our studies suggested that CCND1 was a novel target gene of miR-194 in GC, and miR-194 may inhibit GC cell growth mainly through direct downregulation of CCND1 and induction of cell cycle arrest.

\section{Methods}

\section{Global miRNA expression analysis in TCGA}

The volcano plot data showing the associations between miRNA expression and various clinical characteristics (overall survival, T-stage, pathologic stage and M-stage) of GC patients from TCGA was downloaded from LinkedOmics web tool[12]. The filter conditions were set to $p<0.05$ and event $>300$. MicroRNA-seq data and the correlated detail clinical information of gastric cancer samples were downloaded from The Cancer Genome Atlas (TCGA) by using Sanger Box software developed by ShengXinRen. Expression level of per miRNA was calculated from log2 of its TPM (transcripts per million) value. 


\section{Cell culture and transfection}

The human gastric cancer cell line BGC823 and SGC7901 were purchased from the Shanghai Cell Bank of Chinese Academy of Sciences (Shanghai, China). The two gastric cancer cell lines were cultured in DMEM medium containing $10 \%$ fetal bovine serum, $100 \mathrm{U} / \mathrm{mL}$ penicillin, $100 \mathrm{U} / \mathrm{mL}$ streptomycin and $0.03 \%$ glutamine at $37^{\circ} \mathrm{C}$ in $5 \% \mathrm{CO}_{2}$.

For siRNA transfection, the miR-194 mimics and inhibitors were designed and synthesized by Genepharma (Shanghai, China). For miR-194 mimics, 5'-UGUAACAGCAAC UCCAUGUGGA-3', 5'CACAUGGAGUUGCUGUUACAUU-3'; for miR-194 inhibitors, 5'-UCCACAUGGAGUUGCUGUUACA-3', for si-NC, 5'-UUCUCCGAACGUGUCA CGUTT-3', 5'-ACGUGACACGUUCGGAGAATT-3'; for miRNA inhibitor-NC, 5'CAGUACUUUUGUGUAGUACAA-3'). Two different GC cell lines were seeded into 6-well plates and grown overnight. The next day, when the cell plating density reached $20 \%-30 \%$, GC cells were transfected with siRNAs (final concentration, $50 \mathrm{nM}$ ) by Lipofectamine 2000 (Invitrogen) according to the manufacturer's instructions. At $48 \mathrm{~h}$ post-transfection, cells were harvested for qPCR analysis, flow cytometry assays and RNA sequencing. At $72 \mathrm{~h}$ post-transfection, cells were harvested for western blot.

\section{Cell proliferation assays}

For cell proliferation assays, cells transfected with miRNA mimics or inhibitors for $24 \mathrm{~h}$ were reseeded in 96 -well plates at 2,000 cells/well in a final volume of $100 \mu \mathrm{L}$ and cultured for 4 days. The effects of miR194/CCND1 on cell proliferation were determined with CCK-8 assay every 24 hours. Subsequently, $10 \mu \mathrm{L}$ of CCK-8 solution (Biosharp, China) were added into each well and incubated for 2 hours. Optical density was measured at a wavelength of $490 \mathrm{~nm}$ by an automatic microplate reader (Bio Tek, USA). Triplicate wells were assayed for each experiment, and three independent experiments were performed. Data were expressed as the OD490 mean \pm S.D.

\section{Quantitative RT-PCR}

For RNA extraction, gastric cancer cells were grown in 6-well plates and transfected with siRNAs. After 48 hours, remove the medium and directly add $800 \mu \mathrm{L}$ Trizol into the 6-well plate to harvest samples. Total RNA was extracted using Trizol reagent (Invitrogen, USA) according to the manufacturer's instructions. The isolated RNA was treated with RNase-free DNase I (Roche) for 15-30 minutes as we described before [13]. RNA purity and concentration were checked using the NanoPhotometer spectrophotometer (IMPLEN, CA, USA). PCR was performed to ensure removal of genomic DNA by using RNA samples as templates. Reverse transcription was performed to obtain CDNA by using $1 \mu \mathrm{g}$ total RNA as templates according to the manufacturer's instructions of the PrimeScript ${ }^{T M}$ RT reagent Kit (Perfect Real Time, Takara).

For Quantitative RT-PCR, all the cDNA samples were diluted 5 times as templates of qPCR. The qPCR protocol was using One Step TB Green PrimeScript ${ }^{\text {TM }}$ RT-PCR Kit II (Takara) according to the manufacturer's instructions. The qPCR analysis was conducted on Bio-Rad CFX Manager 3.1 real-time PCR system. The Cycling conditions were as follows: $95^{\circ} \mathrm{C}$ for $30 \mathrm{~s}, 95^{\circ} \mathrm{C}$ for $5 \mathrm{~s}$ and $60^{\circ} \mathrm{C}$ for $30 \mathrm{~s}$. The 
reaction was performed for 40 cycles. The mRNA expression level was determined by using the specific primers (CCND1-F: 5'-TGAACTACCTGGACCGCTTC-3', CCND1-R: 5'-CCACTTGAGCTTGTTCACCA-3'; ACTINF: 5'-ATCGTCCACCGCAAATGCTTCTA-3', ACTIN-R: 5'-AGCCATGCC AATCTCATCT TGTT-3').

\section{Western blot}

After $72 \mathrm{~h}$ transfected with miRNA, Gastric cancer cells were lysed in RIPA buffer added $1 \mathrm{mM}$ PMSF. Approximately $100 \mu \mathrm{g}$ of total protein was electropharesed through $10 \%$ SDS polyacrylamide gels and were then transferred to a PVDF membrane (Millipone). After blocking with $5 \%$ skimmed milk at $4{ }^{\circ} \mathrm{C}$ for 1h, the membrane was incubated with CCND1 antibody (1:1000, Proteintech) and GAPDH (1:1000, Proteintech) at $4^{\circ} \mathrm{C}$ overnights. The blots were then washed and incubated with horseradish peroxidase (HRP)-conjugated secondary antibody (1: 10000, Earthox) for $1.5 \mathrm{~h}$ at room temperature. Detection was performed by using a SuperLumia ECL HRP Substrate Kit (Abbkine). The defined sections of the film were scanned for image capture and quantification using Adobe Photoshop software (Adobe Systems Incorporated, USA) and Image J software (Bio-rad, USA).

\section{Luciferase reporter assay}

For wildtype luciferase reporter vector construction, the 3' untranslated region of CCND1 (from 1900 nt to $2635 \mathrm{nt}$ ) was amplified by PCR and ligated into the pMir-GLO luciferase reporter vector (Promega, USA). For mutant luciferase reporter vector construction, the mutant CCND1 3'UTR (replaced TGTTACA with AAAAACA) were cloned into the MCS region of pMir-GLO luciferase reporter vector with Sac I and Xba I. The primers for wildtype and mutant CCND1 3'UTR were as follows, F: 5'-gggagctcCTGTCCCACTC CTACGATAC-3', R1 (wildtype): 5'-tctctagaTGTAACATCAAAGGCAGAAGG-3', R2 (Mutant): 5'tctctagaTGTTTTTTCAAAGGCAGAAGGTTTGTGT-3'. The luciferase reporter assay was conducted as we previously described before[14]. Briefly, the BGC823 cells were seeded into 12-well-tissue plates $24 \mathrm{~h}$ before transfection, and then co-transfected with $5 \mathrm{nM}$ siRNA and $1 \mathrm{mg}$ plasmid using the Lipofectamine 2000 Reagent (Invitrogen), according to the manufacturer's instructions. After another 48h, cells were assayed using the Dual-Luciferase reporter assay system kit (GeneCopoeia, USA). All experiments were performed in triplicate and data were pooled from three independent experiments.

\section{Flow cytometry assays}

After 48h transfected with miR-194 mimics and corresponding negative control siRNAs, SGC7901 and BGC823 cells were collected and stained with propidium iodide (BB-4104, BestBio, CHINA) and performed in accordance with the manufacture's protocol. Cell cycle analysis was performed on the CytoFLEX machine (Beckman, USA). The cell cycle distribution was quantified using the CytExpert software.

\section{RNA sequencing}

After $48 \mathrm{~h}$ transfected with miRNA mimics and corresponding negative control siRNAs, SGC7901 and BGC823 cells total RNA was extracted to perform RNA sequencing. A total amount of $1.5 \mu \mathrm{g}$ RNA per sample was used as input material for the RNA sample preparations. The whole step of library 
construction and sequencing was performed at Shanghai Lifegenes Technology Co., Ltd. Random hexamer primer cDNA libraries were sequenced on Illumina Hiseq 4000 sequencing platform (Illumina) according to the manufacturer's instructions for paired-end 150 bp reads (Lifegenes, Shanghai). The RNAseq data was uploaded on the GEO section of NCBI web server. The gene expression omnibus accession number is GSE134308.

\section{Statistical analysis}

Data from at least three independent experiments performed in triplicate are presented as the mean \pm S.D. Comparisons were performed using the Student's paired t-test, Spearman's correlation test; $P<0.05$ was considered statistically significant.

\section{Results}

\section{Identification of the miRNAs associated with clinical outcome of gastric cancer patients.}

The association between miRNAs expression patterns and clinical outcomes was analyzed using LinkedOmics database, which are web-based tools to deliver fast and customizable functionalities based on The Cancer Genome Atlas (TCGA) data[12]. The significances (- $\log _{10} P$ value) of the correlation between the expression of all microRNAs and the overall survival, T stage (or pathological stage), and $\mathrm{M}$ stage of GC were shown in the four Volcano Plots. The results showed that GC patients with higher expression of miR-100 (miR-653, miR-125a, miR-218, etc.) or lower expression of miR-182 (miR-7, miR-96, miR-15b, miR-194, etc.) usually have a shorter overall survival time $(p<0.05$, Fig 1 A). The expression of miR-217 (miR-181a, miR-132, miR-100, etc.) was positively correlated with the T stage of GC, while the expression of miR-320a (miR-7, miR-182, miR-183, miR-194, etc.) was negatively correlated with the $T$ stage of GC ( $p<0.05$, Fig 1B). The expression of miR-130a (miR-217, miR-132, miR-100, etc.) was positively related to the pathological stage of GC, while the expression of miR-7 (miR-320a, miR-182, miR183 , miR-194, etc.) was negatively related to the pathological stage of $\mathrm{GC}(\mathrm{p}<0.05$, Fig $1 \mathrm{C})$. In addition, we also note that GC tissues in M1 stage usually possessed higher expression of miR-152 (miR-181a, miR-125b, miR-100, etc.) and lower expression of miR-194 and miR-147b $(p<0.05$, Fig $1 \mathrm{D})$ compared to the GC tissues in M0 stage.

To determine the most appropriate diagnostic and prognostic biomarkers for $\mathrm{GC}$, we plotted Venn diagrams based on the number of miRNAs that significantly positively associated with overall survival rate, $\mathrm{T}$ stage, pathological stage and $\mathrm{M}$ stage. Table S1 lists miRNAs that are significantly related to at least two clinical characteristics (such as survival rate, $T$ stage, pathological stage, and $M$ stage). As shown in Figure 1E, F and Table S1, miR-100, miR-125b, miR-199a and miR-194 were the 4 most promising biomarkers that can accurately predict cancer stage and reflect an individual's cancer risk in GC. Additionally, our data also suggested that miR-100, miR-125b, miR-199a were unfavorable prognostic biomarkers in GC, while miR-194 was favorable prognostic biomarker in GC. 
In order to better understand the correlation between miR-194 expression and clinical pathology of gastric cancer, we further analyzed the RNA-Seq data of miR-194 in the stomach cancer tissues from TCGA database $(n>375)$. The results showed that miR-194 expression level in the diffuse type GC was significantly lower than that in the intestinal type GC (Fig 2A, p < 0.0001). Moreover, miR-194 expression tends to be higher in moderately or highly differentiated gastric cancer tissues than that in poorly differentiated gastric cancer tissues (Fig 2B, $p<0.01$ ).

Furthermore, there was a significant positive correlation between miR-194 expression and the extent of GC progression (Fig 2C). Similarly, miR-194 was more highly expressed in the gastric mucosa (T1) than tumors extending beyond the gastric mucosa layer (T2+T3+T4) (Fig 2D). Patients with lower expression of miR-194 were more likely to have distant tumor metastasis (Fig 2E). More importantly, patients with lower miR-194 expression tended to have a shorter overall survival time than those patients with higher miR-194 expression (Fig 2F). Collectively, these results together suggested that miR-194 may be tumor suppressor in GC and miR-194 could serve as an independent diagnostic and prognostic biomarker for GC.

\section{The preferences of miR-194-5p in regulating target genes}

To figure out the preference of miR-194 in regulating target genes, RNA sequencing studies was performed in the two gastric cancer cell lines (SGC7901 and BGC823) that transfected with miR-194-5p mimics and corresponding negative control siRNAs. The RNA sequencing results were uploaded on the NCBI website. And the gene expression omnibus accession number was GSE134308. The heat map showed that there were hundreds of coding genes (non-coding genes were not included in this study) have altered their expression levels after treatment with miR-194-5p mimics (Fig 3A, $|F C|>1.5$ ). Of these, approximately 138 coding genes were relatively strongly downregulated by miR-194 mimics, while almost 70 coding genes that relatively severely upregulated by miR-194 mimics in both SGC7901 and BGC823 cell lines (Fig 3B). And the top 23 coding genes that most strongly downregulated by miR-194 were listed in the Table $1(\log 2 \mathrm{FC}<-0.9)$.

According to the binding sequence differences in the miR-194-5p seed region, the miR-194-5p binding sites in mRNAs can be divided into three types, including 7-mer-A1, 7-mer-m8 and 8-mer (Fig 3C). Then, we annotated the information of miR-194 binding sites in the coding genes that downregulated by miR194 using miRcode web-based tools. The results showed that nearly $60 \%$ of the coding genes contained miR-194-5p binding sites (Fig 3D). And the distribution proportion of the three binding modes is very close, suggested that any type of binding manner between miR-194 and mRNAs would be effective (Fig $3 \mathrm{E})$. Next, we further analyzed the binding location preference of miR-194 in mRNA sequence. The results showed that almost $86 \%$ of miR-194 binding sites were located at 3 ' untranslated region of target genes (Fig 3F). In addition, no significant correlation was observed between expression alteration $\left(\log _{2} \mathrm{FC}\right)$ and the number of miR-194 binding sites in both SGC7901 and BGC823 cell lines (Fig 3G and H).

MiR-194 negatively regulated CCND1 expression by binding on the 3 ' untranslated region in GC. 
Next, we conducted KEGG pathway analysis to determine the most affected pathways by miR-194 in GC. As shown in Supplementary Figure, the cell cycle pathway was one of the most affected pathways by miR-194. RNA-seq data showed that CCND1 was strongly downregulated in both SGC7901 and BGC823 cell lines (log2FC value was -0.84 in SGC7901, -0.71 in BGC823). To confirm the RNA-seq results, we investigated the CCND1 expression in two GC cell lines after treatment with miR-194 mimics and inhibitors by qPCR analysis and the western blot assay. The results showed that miR-194 overexpression significantly decreased CCND1 expression in GC, while miR-194 inhibitor significantly increased CCND1 expression in GC (Fig 4A-D). These results strongly indicated that CCND1 was negatively regulated by miR-194.

Based on the above results, we further analyzed the information of miR-194 binding site in the CCND1 transcript using the miRcode web-based tools and predicts the folding energy between miR-194 and CCND1 transcript using RNA22 web-based tools $[15,16]$. As shown in Figure 5A, in the 3' untranslated region of CCND1, there was only one miR-194 binding site (7-mer-A1). The folding energy between miR194 and CCND1 transcript was $-9 \mathrm{Kcal} / \mathrm{mol}$, suggested that the negative regulation of miR-194 on CCND1 expression might be through regulating mRNA stability by the direct binding on CCND1 transcripts. To further validate this hypothesis, luciferase reporter assay was performed. Since the length of $3^{\prime}$ untranslated region of CCND1 was too large to be amplified, we selected to clone the 735-length (from $1900 \mathrm{nt}$ to $2642 \mathrm{nt}$ ) into the luciferase reporter vector with original sequence (wildtype) or with the $4 \mathrm{nt}$ changed (mutant) in the miR-194 binding site (Fig 5B). As expected, compared to the BGC823 cells that transfected with negative control siRNA and wildtype luciferase vector, a significant decrease and increase was observed in the BGC823 cells that transfected with miR-194 mimics and inhibitors, respectively. However, no obvious changes were detected in the BGC823 cells that transfected with mutant CCND1 3'UTR luciferase vector (Figure 5C). These results strongly suggested that miR-194 negatively regulate CCND1 expression at post-transcriptional level.

\section{MiR-194 suppressed GC cell proliferation through induction of GC cell cycle arrest.}

The cyclin protein CCND1 plays an essential role in the cell cycle process. Increasing studies have demonstrated that downregulation of CCND1 induces G1 phase arrest and then impairs cell growth[17]. Therefore, we performed the cell proliferation assay in SGC7901 and BGC823 cells that transfected with miR-194 mimics and inhibitors. The results showed that miR-194 mimics markedly suppressed the cell proliferation in GC, while miR-194 inhibitors slightly promoted the cell proliferation in GC (Fig 6 A and B). On the other hand, we conducted the flow cytometry assays in the two GC cell lines after treatment with miR-194 mimics and corresponding negative control siRNAs. The flow cytometry analysis showed that compared to NC group, miR-194 significantly decreased the number of the sub G0 phase and $\mathrm{S}$ phase cells, but increased the number of G1 phase cells in both SGC7901 and BGC823(Fig 6 C-F). These results together suggested that miR-194 inhibits GC cell growth via induction of cell cycle arrest.

\section{Discussion}


Gastric cancer, a leading cause of cancer-related deaths, is a heterogeneous disease with many kinds of subtypes. Mountain evidences show that different subtype of gastric cancers usually possess distinct clinical outcomes $[18,19]$. In the past, only the coding genes were developed as biomarkers for tumor diagnosis. However, nowadays increasing non-coding RNAs are found to be functional in multiple biological process. And more and more non-coding genes have been developed as biomarkers for tumor diagnosis. As a member of non-coding RNAs, miRNAs usually have specific expression profiles in cancer cells and tissues, and can enter the body fluid circulation. Therefore, the development of miRNAs as diagnostic markers is gaining more and more attention.

To develop biomarkers that can accurately predict stomach cancer stage and reflect an individual's stomach cancer risk, miRNA expression analysis was performed using the available TCGA data. And our global miRNA expression analysis (RNA-seq data of 391 GC patients) identifies miR-194, miR-100, miR125b and miR-199a as the 4 most promising biomarkers for gastric cancer. 3 of them (miR-100, miR-125b and miR-199a) have also identified by another independent research using microRNA microarray data of 353 GC samples (from 182 GC patients), suggested our global miRNA expression analysis results are convincing[20].

Although the role of miR-194 in gastric cancer remains controversial, our findings and most of the previous studies tend to think that miR-194 plays a tumor suppressor role in GC. The expression pattern and function of miR-100, miR-125b and miR-199a in GC have widely been studied. Wu et al. have reported that miR-125b promotes GC progression by targeting PPP1CA-Rb signal pathways, and predicts a poor prognosis in GC[21]. Another study by Sui et al. reported that miR-125b is associated with poor prognosis and trastuzumab resistance in HER2-positive gastric cancer. Chang et al. demonstrated that miR-125b promotes invasion and metastasis of gastric cancer by targeting STARD13 and NEU1. Yang et al. proved that miR-100 upregulation in GC play critical roles in primary human gastric tumorigenesis and progression [22, 23]. Similarly, miR-199b have also been reported to play oncogenic roles in GC [24-26].

As mentioned in the Introduction section, miRNAs usually have preferences in the regulation of their target genes. Therefore, our next tasks are to figure out the preference of miR-194 in regulating target genes and to elucidate the molecular mechanisms by which miR-194 inhibits GC. RNA sequencing studies in two GC cell lines showed that miR-194 tends to regulate target genes by binding on the 3'UTR in a 7-mer-A1 or 7-mer-m8 or 8-mer manner. Nearly 138 coding genes are strongly downregulated by miR194 in both SGC7901 and BGC823 GC cell lines, including CCND1. Previous studies have showed that miR-194 inhibited tumor progression by downregulating FOXM1[8], RBX1[9] and KDM5B[27]. Interestingly, after analysis of the miR-194 binding sites in those genes, we note that all the genes except FOXM1 contained miR-194 binding sites (7-mer-A1: KDM5B; 7-mer-m8: RBX1, KDM5B). Correspondingly, RBX1 and KDM5B were indeed slightly downregulated, while FOXM1 expression almost has not alteration after treatment with miR-194 mimics (data not shown). Therefore, we think miR-194-RBX1 and miR-194KDM5B axis might not be at least the primary pathways mediated by miR-194 in GC inhibition. 
To our knowledge, our study provided the first comprehensive transcriptome analysis about miR-194 in GC. The RNA-seq analysis showed that CCND1 was one of the most downregulated genes by miR-194 in GC. The KEGG pathway analysis showed that cell cycle was one of the most affected pathways by miR194 in GC. CCND1 was proved to be a novel target gene of miR-194 in GC. Therefore, our finding suggested that miR-194 inhibits the progression of GC mainly through affecting the cell cycle pathway.

In addition, the qRT-PCR assay, western blot assay and luciferase reporter assay together suggested that miR-194 negatively regulated CCND1 expression by binding on its 3'UTR region. Mounting evidences showed that the downregulation of CCND1 by miRNAs, such as miR-155[17], miR-490-5p[28], miR193b[29], etc., would simultaneously induce cell cycle arrest at G1 phase. Consistent with previous reports, our findings also showed that miR-194 inhibits GC cell growth and induces cell cycle arrest at G1 phase. Previous published studies reported that lower expression of miR-194 tended to have larger tumor size [7]. It's well known that cell cycle process was closely related to cancer cell growth. Given this, our results may at least partially provide a possible explanation about this phenomenon.

\section{Conclusion}

This study provides a comprehensive miRNA expression analysis in GC using the TCGA data. MiR-100, miR-125b, miR-199a and miR-194 are identified to be the 4 most promising miRNAs that can serve as prognostic and diagnostic biomarkers for GC. Additionally, our findings would at least partially clarify this controversy regarding the role of miR-194 in GC. MiR-194 suppresses GC cell growth mainly through targeting CCND1 and induction of cell cycle arrest.

\section{Abbreviations}

GC: Gastric cancer

TCGA: The Cancer Genome Atlas

KEGG: Kyoto Encyclopedia of Genes and Genomes

UTR: Untranslated region

EMT: epithelial mesenchymal transition

TPM: transcripts per million

qRT-PCR: quantitative reverse transcription Polymerase Chain Reaction

GEO: Gene Expression Omnibus

\section{Declarations}


Availability of data and materials

The datasets generated during and/or analysed during the current study are available in the GEO repository, [https://www.ncbi.nlm.nih.gov/geo/query/acc.cgi?acc=GSE134308].

\section{Acknowledgements}

We are very grateful to Dr. JiWei Li for contributing to the RNA-Seq analysis.

\section{Funding}

This study was supported by grants from the National Natural Science Foundation of China (grant no. 81802375); the Natural Science Foundation of Hubei Provincial Department of Education (grant nos. Q20182103); the Cultivating Project for Young Scholar at Hubei University of Medicine (grant nos. 2016QDJZR07, 2017QDJZR05 and 2016QDJZR17).

\section{Author information}

Laboratory of tumor biology, School of Basic Medical Sciences, Hubei University of Medicine, Shiyan, Hubei 442000, P.R, China

Dandan Li, Jingjie Wang, Meixin Zhang, Xinhui Hu, Jiajun She, Mengyu Cai, Lijuan Yang, Weijia Liu, Xuemei Qiu and Shanshan Qin

School of Biomedical Engineering, Hubei University of Medicine, Shiyan, Hubei 442000, P.R, China

Dandan Li, Jingjie Wang and Shanshan Qin

Hubei Key Laboratory of Wudang Local Chinese Medicine Research, Hubei University of Medicine, Shiyan, Hubei 442000, P.R. China

Shanshan Qin

\section{Contributions}

QS conceived and designed the study. QS wrote the paper. LD performed most of the experiments. WJ, $Z M, H X, S J, C M, Y L, L W, H Q$ and QX carried out initial data analyses and performed partial of the experiments. All authors contributed to drafting the manuscript. All authors have read and approved the final submitted manuscript.

\section{Corresponding authors}

Correspondence to Dr. Shanshan Qin.

\section{Ethics declarations}


Ethics approval and consent to participate

Not applicable.

Consent for publication

Not applicable.

Competing interests

The authors declare that they have no competing interests

Additional information

Publisher's Note

Springer Nature remains neutral with regard to jurisdictional claims in published maps and institutional affiliations.

\section{References}

1. Shah S, Underwood FE, Ng WK, Chan WY, Castaneda D, Riyat N, Azhari H, Ng SC, Kaplan G: The Global Incidence of Gastric Cancer in the 21st Century: A Systematic Review of Population-Based Studies from 2007-2017. Gastroenterology 2018, 154(6):S986-S986.

2. Sano T: Gastric cancer: Asia and the world. Gastric Cancer 2017, 20:S1-S2.

3. Yusefi AR, Bagheri Lankarani K, Bastani P, Radinmanesh M, Kavosi Z: Risk Factors for Gastric Cancer: A Systematic Review. Asian Pacific journal of cancer prevention : APJCP 2018, 19(3):591603.

4. Ling $\mathrm{H}$, Fabbri $\mathrm{M}$, Calin GA: MicroRNAs and other non-coding RNAs as targets for anticancer drug development. Nature reviews Drug discovery 2013, 12(11):847.

5. Reddy KB: MicroRNA (miRNA) in cancer. Cancer cell international 2015, 15(1):38.

6. Ferracin M, Laprovitera N, Porcellini E, Grzes M: Cancer site-specific multiple microRNA quantification by droplet digital PCR. Frontiers in oncology 2018, 8:447.

7. Song Y, Zhao F, Wang Z, Liu Z, Chiang Y, Xu Y, Gao P, Xu H: Inverse association between miR-194 expression and tumor invasion in gastric cancer. Ann Surg Oncol 2012, 19 Suppl 3:S509-517.

8. Li Z, Ying X, Chen H, Ye P, Shen Y, Pan W, Zhang L: MicroRNA-194 Inhibits the EpithelialMesenchymal Transition in Gastric Cancer Cells by Targeting FoxM1. Digestive Diseases \& Sciences 2014, 59(9):2145-2152.

9. Chen X, Wang Y, Zang W, Du Y, Li M, Zhao G: miR-194 targets RBX1 gene to modulate proliferation and migration of gastric cancer cells. Tumor Biology 2015, 36(4):2393-2401. 
10. Bao J, Zou JH, Li CY, Zheng GQ: miR-194 inhibits gastric cancer cell proliferation and tumorigenesis by targeting KDM5B. European review for medical and pharmacological sciences 2016, 20(21):44874493.

11. Peng Y, Zhang X, Ma Q, Yan R, Qin Y, Zhao Y, Cheng Y, Yang M, Wang Q, Feng X: MiRNA-194 activates the Wnt/ $\beta$-catenin signaling pathway in gastric cancer by targeting the negative Wnt regulator, SUFU. Cancer Lett 2017, 385:117-127.

12. Vasaikar SV, Straub P, Wang J, Zhang B: LinkedOmics: analyzing multi-omics data within and across 32 cancer types. Nucleic Acids Res 2018, 46(D1):D956-D963.

13. Qin SS, Tang YH, Chen YP, Wu PZ, Li MR, Wu GJ, Jiang HW: Overexpression of the Starch Phosphorylase-Like Gene (PHO3) in Lotus japonicus has a Profound Effect on the Growth of Plants and Reduction of Transitory Starch Accumulation. Front Plant Sci 2016, 7.

14. Li D, Cheng P, Wang J, Qiu X, Zhang X, Xu L, Liu Y, Qin S: IRF6 is directly regulated by ZEB1 and ELF3, and predicts a favorable prognosis in gastric cancer. Frontiers in oncology 2019, 9:220.

15. Jeggari A, Marks DS, Larsson E: miRcode: a map of putative microRNA target sites in the long noncoding transcriptome. Bioinformatics 2012, 28(15):2062-2063.

16. Hofacker IL, Fontana W, Stadler PF, Bonhoeffer LS, Schuster P: Fast Folding and Comparison of RNA Secondary Structures. Monatsh Chem 1994, 125(2):167-188.

17. Ma Z, Ma Y, Xia Q, Li Y, Li R, Chang W, Chen J, Leng Z, Tao K: MicroRNA-155 expression inversely correlates with pathologic stage of gastric cancer and it inhibits gastric cancer cell growth by targeting cyclin D1. Journal of cancer research and clinical oncology 2016, 142(6):1201-1212.

18. Abbas M, Faggian A, Sintali DN, Khan GJ, Naeem S, Shi M, Chen D: Current and future biomarkers in gastric cancer. Biomed Pharmacother 2018.

19. Yasui W, Oue N, Aung PP, Matsumura S, Shutoh M, Nakayama H: Molecular-pathological prognostic factors of gastric cancer: a review. Gastric Cancer 2005, 8(2):86-94.

20. Ueda T, Volinia S, Okumura H, Shimizu M, Taccioli C, Rossi S, Alder H, Liu C-g, Oue N, Yasui W: Relation between microRNA expression and progression and prognosis of gastric cancer: a microRNA expression analysis. The lancet oncology 2010, 11(2):136-146.

21. Wu J-G, Wang J-J, Jiang X, Lan J-P, He X-J, Wang H-J, Ma Y-Y, Xia Y-J, Ru G-Q, Ma J: MiR-125b promotes cell migration and invasion by targeting PPP1CA-Rb signal pathways in gastric cancer, resulting in a poor prognosis. Gastric Cancer 2015, 18(4):729-739.

22. Yang $G$, Gong $Y$, Wang $Q$, Wang $Y$, Zhang $X$ : The role of miR-100-mediated Notch pathway in apoptosis of gastric tumor cells. Cell Signal 2015, 27(6):1087-1101.

23. Yang G, Gong Y, Wang Q, Wang L, Zhang X: miR-100 antagonism triggers apoptosis by inhibiting ubiquitination-mediated p53 degradation. Oncogene 2017, 36(8):1023.

24. Song G, Zeng H, Li J, Xiao L, He Y, Tang Y, Li Y: miR-199a regulates the tumor suppressor mitogenactivated protein kinase kinase kinase 11 in gastric cancer. Biological and Pharmaceutical Bulletin 2010, 33(11):1822-1827. 
25. He X-J, Ma Y-Y, Yu S, Jiang X-T, Lu Y-D, Tao L, Wang H-P, Hu Z-M, Tao H-Q: Up-regulated miR-199a-5p in gastric cancer functions as an oncogene and targets klotho. Bmc Cancer 2014, 14(1):218.

26. Wang Z, Ma X, Cai Q, Wang X, Yu B, Cai Q, Liu B, Zhu Z, Li C: MiR-199a-3p promotes gastric cancer progression by targeting ZHX1. FEBS letters 2014, 588(23):4504-4512.

27. Bao J, Zou J, Li C, Zheng G: miR-194 inhibits gastric cancer cell proliferation and tumorigenesis by targeting KDM5B. European review for medical and pharmacological sciences 2016, 20(21):44874493.

28. Zhao L, Tang XP, Luo RG, Duan J, Wang YC, Yang BB: MicroRNA-490-5P Targets CCND1 to Suppress Cellular Proliferation in Glioma Cells and Tissue Through Cell Cycle Arrest. Curr Neurovasc Res 2018, 15(3):246-255.

29. Wang L, Zhang Y, Zhao L, Liu S, Yu S, Ma Y, Sun G: MicroRNA-193b inhibits the proliferation, migration and invasion of gastric cancer cells via targeting cyclin D1. Acta histochemica 2016, 118(4):323-330.

\section{Tables}

Table 1. The top 23 coding genes that most downregulated by miR-194-5p mimics in gastric cancer. 


\begin{tabular}{|c|c|c|c|c|c|}
\hline \multirow[b]{2}{*}{$\begin{array}{l}\text { Gene } \\
\text { symbols }\end{array}$} & \multirow{2}{*}{$\begin{array}{l}\text { SGC7901 } \\
(\log 2 F C)\end{array}$} & \multirow{2}{*}{$\begin{array}{l}\text { BGC823 } \\
(\log 2 F C)\end{array}$} & \multicolumn{3}{|c|}{ miR-194 binding sites of transcripts } \\
\hline & & & Number & Type & Position \\
\hline ARL6IP5 & -1.201 & -1.093 & 3 & 7-mer-A1 & 3'UTR \\
\hline ATP11C & -1.240 & -1.076 & 2 & 7-mer-A1; 8-mer & $\begin{array}{l}\text { CDS (1); 3'UTR } \\
\text { (1) }\end{array}$ \\
\hline ATP6V1F & -1.160 & -1.051 & 1 & 7-mer-A1 & 3'UTR \\
\hline BTF3L4 & -1.173 & -0.980 & 3 & 7-mer-A1 (2); 7-mer-m8 (1) & 3'UTR \\
\hline CFL2 & -1.221 & -1.147 & 2 & 7-mer-m8 & 3'UTR \\
\hline DUSP9 & -1.358 & -0.965 & 1 & 7-mer-m8 & 3'UTR \\
\hline ERGIC2 & -1.014 & -0.831 & 1 & 8-mer & 3'UTR \\
\hline FZD6 & -0.901 & -0.820 & 2 & 7-mer-A1; 8-mer & $\begin{array}{l}\text { CDS (1); 3'UTR } \\
\text { (1) }\end{array}$ \\
\hline GYG1 & -0.930 & -0.996 & 1 & 8-mer & 3'UTR \\
\hline IL6ST & -1.521 & -1.180 & 4 & 7-mer-A1 (3); 8-mer (1) & 3'UTR \\
\hline PPP1R14B & -1.259 & -1.143 & 1 & 8-mer & CDS \\
\hline GNS & -0.901 & -1.291 & 1 & 8-mer & 3'UTR \\
\hline RETREG1 & -1.151 & -0.977 & 2 & 7-mer-m8 & $\begin{array}{l}\text { CDS (1); 3'UTR } \\
(1)\end{array}$ \\
\hline SDCBP & -1.569 & -1.542 & 1 & 8-mer & 3'UTR \\
\hline PRKAR1A & -1.151 & -1.002 & 1 & 8-mer & 3'UTR \\
\hline GOT2 & -0.926 & -1.044 & 1 & 8-mer & 3'UTR \\
\hline SLC7A5 & -1.029 & -1.339 & 1 & 8-mer & 3'UTR \\
\hline ELOVL3 & -1.387 & -1.371 & 1 & 7-mer-m8 & CDS \\
\hline SRGN & -1.356 & -1.174 & 1 & 8-mer & 3'UTR \\
\hline TCEAL4 & -0.913 & -1.080 & 1 & 8-mer & 3'UTR \\
\hline TEX30 & -1.019 & -1.033 & 2 & 7-mer-A1 (3); 8-mer (1) & 3'UTR \\
\hline TMED5 & -1.146 & -1.224 & 6 & $\begin{array}{l}\text { 7-mer-A1 (3); 7-mer-m8 (2) } ₫ 8 \text { - } \\
\text { mer (1) }\end{array}$ & 3'UTR \\
\hline YWHAZ & -1.098 & -0.951 & 1 & 7-mer-m8 & 3'UTR \\
\hline
\end{tabular}


Figures

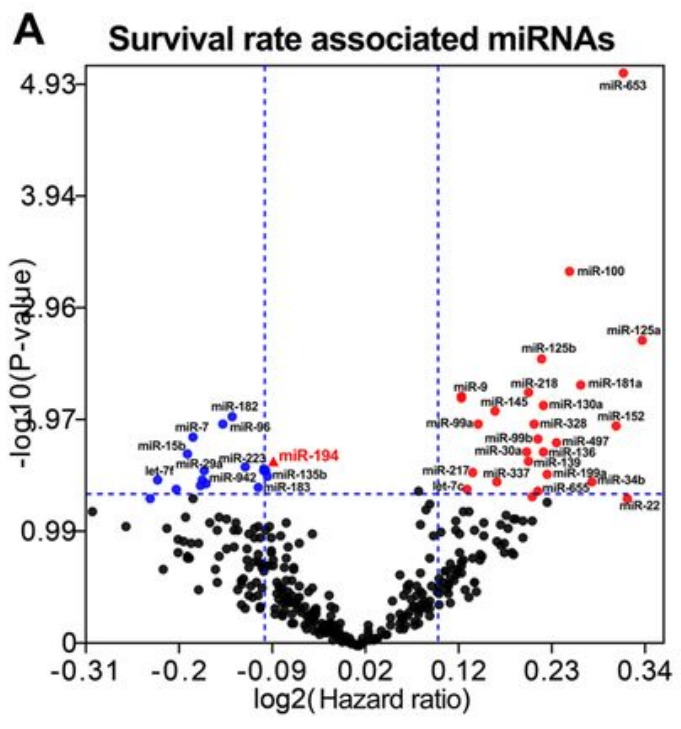

C

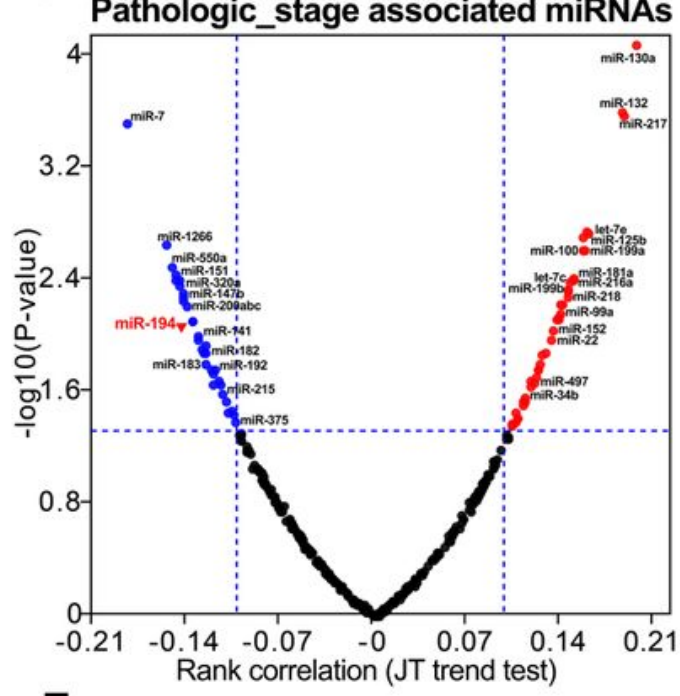

E

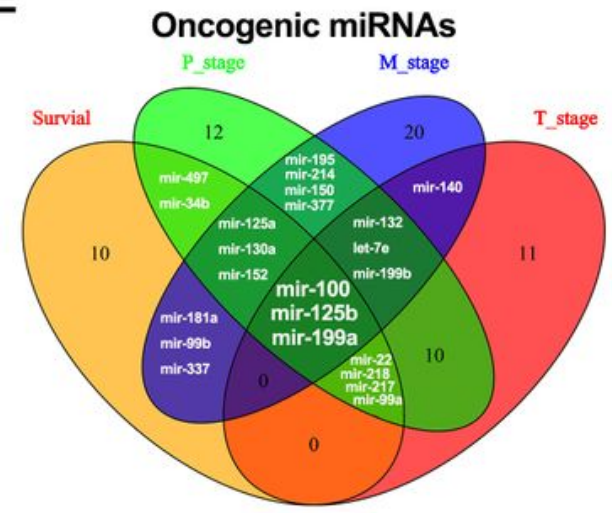

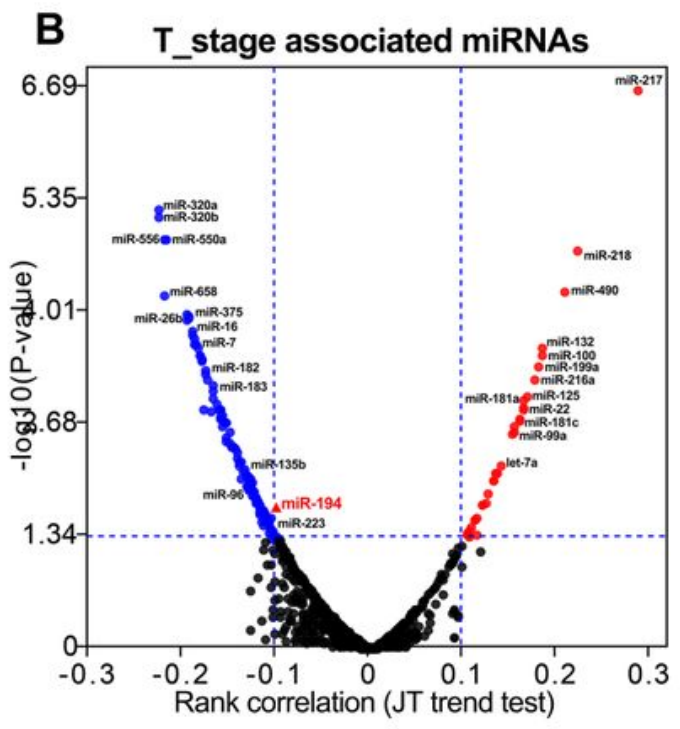

D M_stage associated miRNAs

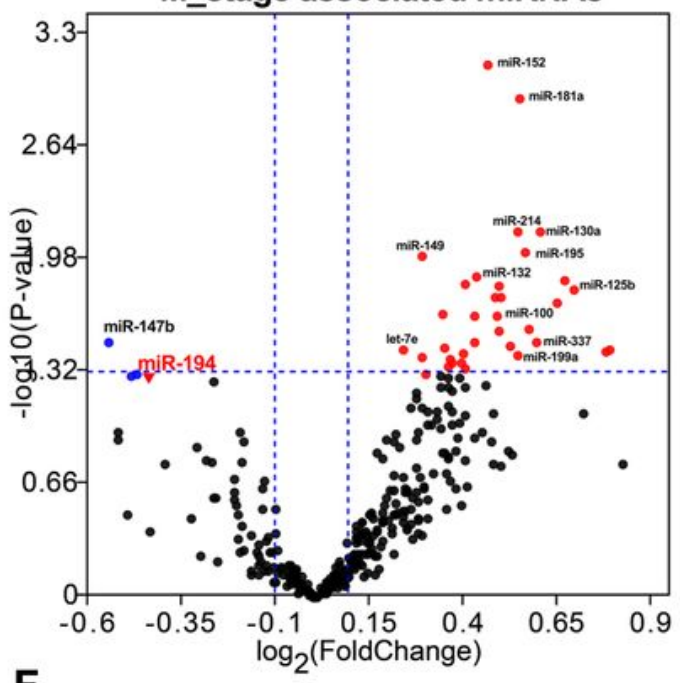

F

Tumor supressor miRNAs

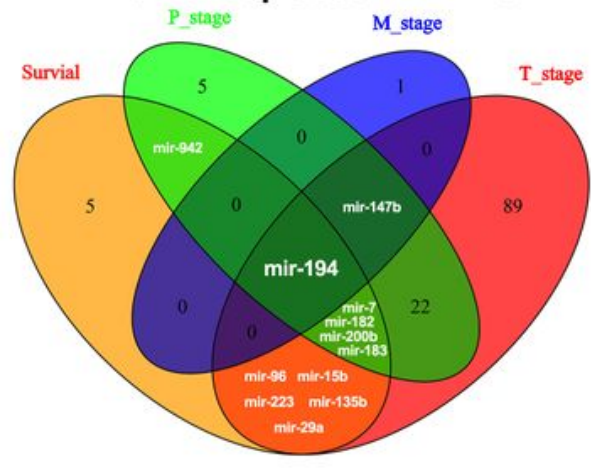

Figure 1 
The miRNAs that showed significant associations with clinical characteristics and outcomes of GC cohorts from TCGA. (A) The volcano plot displays miRNAs that significantly associated with survival rate in the GC cohorts from TCGA. The red dot means the miRNAs that significantly negatively correlated to the survival time; the blue dot means the miRNAs that significantly positively correlated to the survival time $(p<0.05)$. (B) The volcano plot displays miRNAs that significantly associated with $T$ stage of the GC cohorts from TCGA. The red dot means the miRNAs that significantly positively correlated to the T stage; the blue dot means the miRNAs that significantly negatively correlated to the T stage $(p<0.05)$. (C) The volcano plot displays miRNAs that significantly associated with pathological stage of the GC cohorts from TCGA. The red dot means the miRNAs that significantly positively correlated to the pathological stage; the blue dot means the miRNAs that significantly negatively correlated to the pathological stage ( $p$ < 0.05); (D) The volcano plot displays miRNAs that significantly associated with $\mathrm{M}$ stage of the GC cohorts from TCGA. The red dot means the miRNAs that significantly positively correlated to the $\mathrm{M}$ stage; the blue dot means the miRNAs that significantly negatively correlated to the M stage $(p<0.05)$; $(E)$ The Venn diagram displays miRNAs that predicts poor prognosis in $G C(p<0.05)$. ( $F)$ The Venn diagram displays miRNAs that predicts favorable prognosis in GC $(p<0.05)$.

A

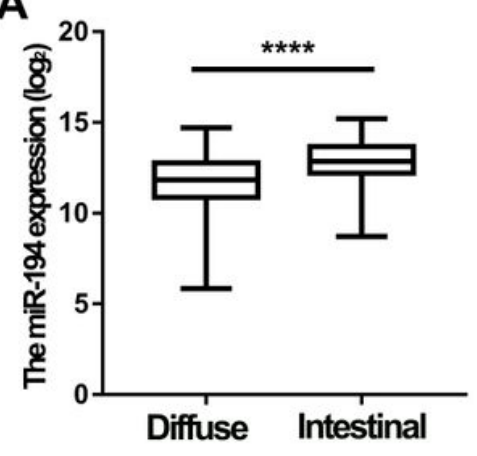

D

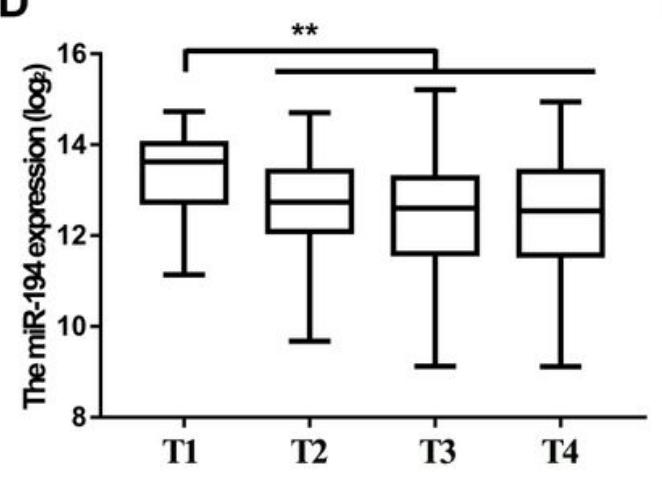

B

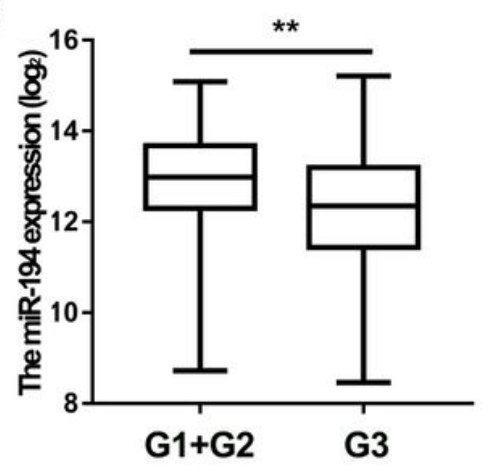

E

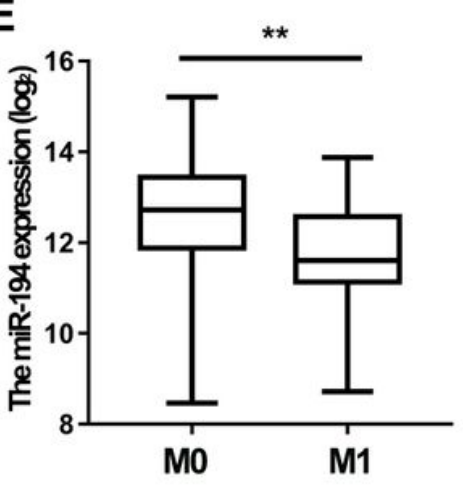

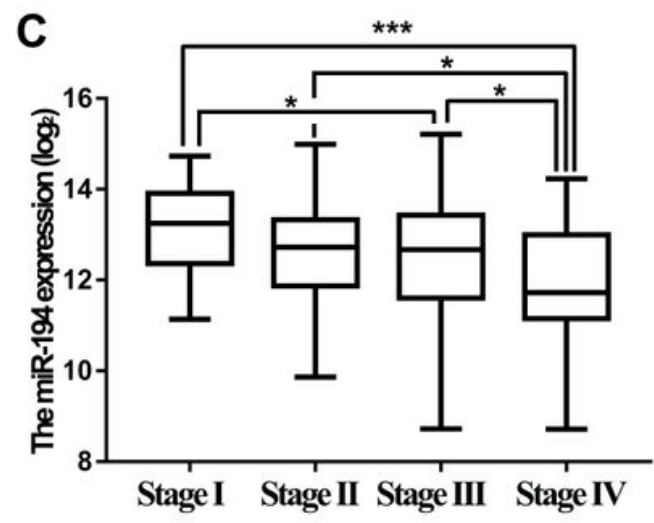

$\mathbf{F}$

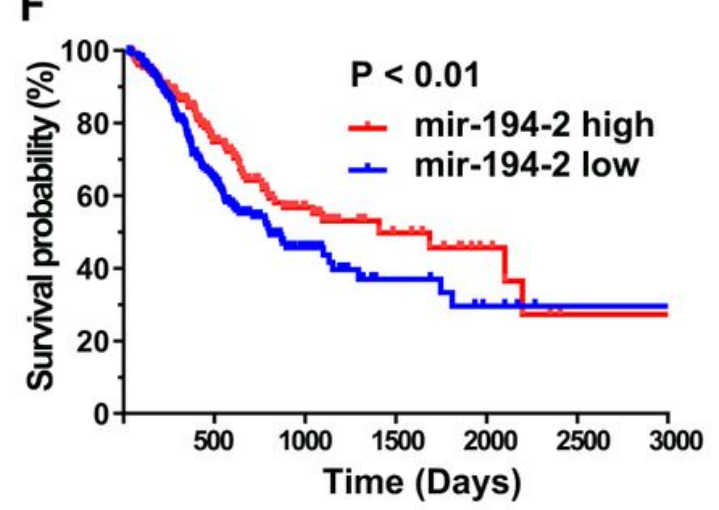

Figure 2

The correlations between miR-194 expression level and clinicopathological features of the GC patients from TCGA. (A) Difference in expression levels of miR-194 between intestinal and diffuse GC tissues; (BE): The expression level of miR-194 in different differentiation grade (B) Pathological stages (C), T stages (D), and M-stages (E) of GC tissues from TCGA; (F) Kaplan-Meier analysis of the overall survival time 
differences between GC patients with relative higher miR-194 expression and GC patients with relative lower miR-194 expression (****, $\mathrm{P}<0.0001$; ***, $\mathrm{P}<0.001$; **, $\mathrm{P}<0.01$; *, $\mathrm{P}<0.05$ )
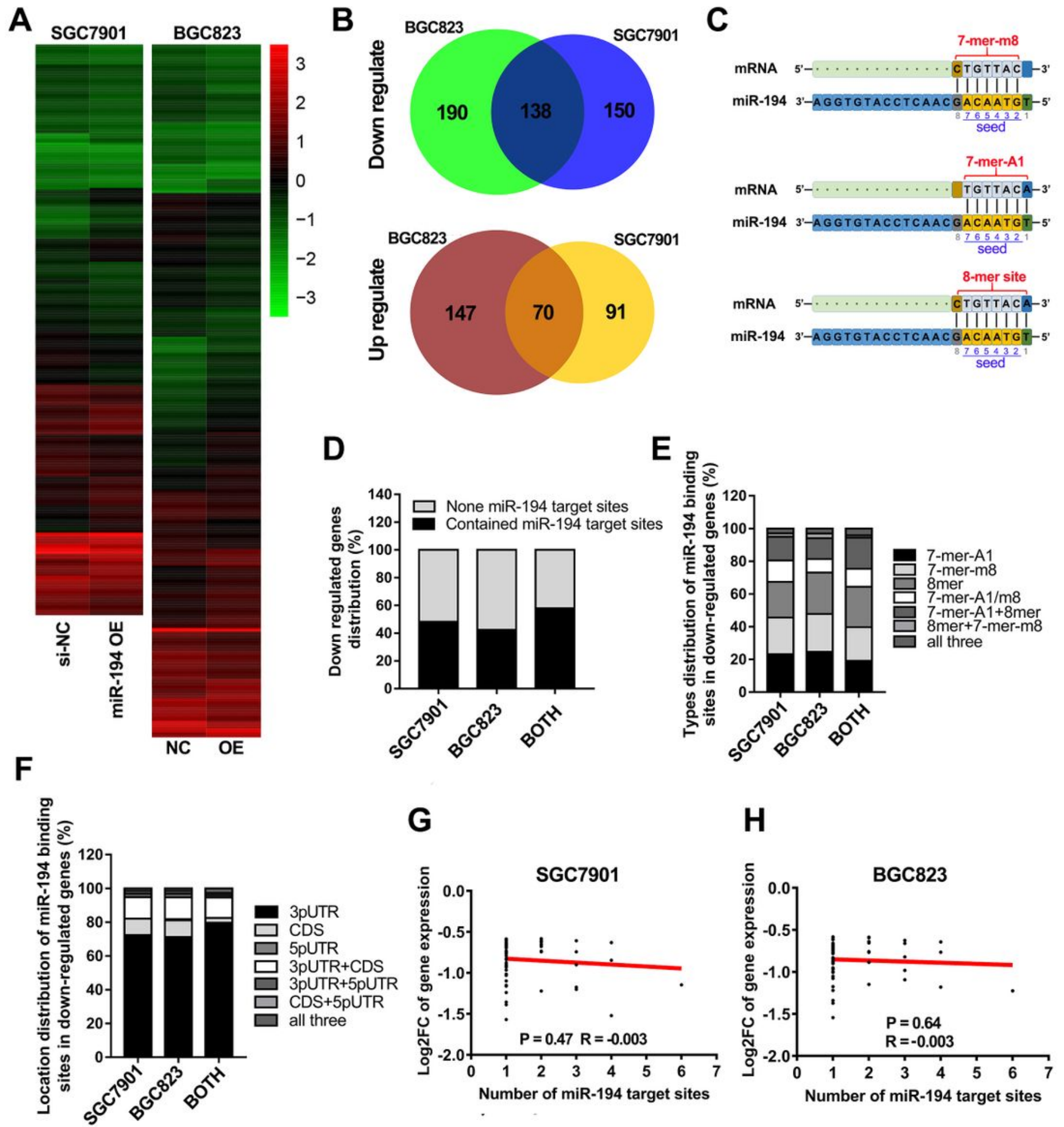

\section{Figure 3}

The preferences of miR-194 in regulating target genes in GC. (A) The heatmap of coding genes that regulated by miR-194 in SGC7901 and BGC823 (FC > 1.5); (B) The coding genes that downregulated or upregulated by miR-194 in both SGC7901 and BGC823 cells; (C) The binding mode between coding gene 
transcripts and miR-194 seed region; (D) The percentage of gens that contain none miR-194 binding site among those genes that downregulated in both SGC7901 and BGC823 cells; (E) The distribution of different binding modes between miR-194 and target transcripts; $(F)$ The distribution of different binding regions between miR-194 and target transcripts; $(\mathrm{G}$ and $\mathrm{H})$ : The correlation between the FC value of target gene expression and the number of miR-194 binding sites in target genes by analysis the RNA-seq data of SGC7901 (G) and BGC823 (H).
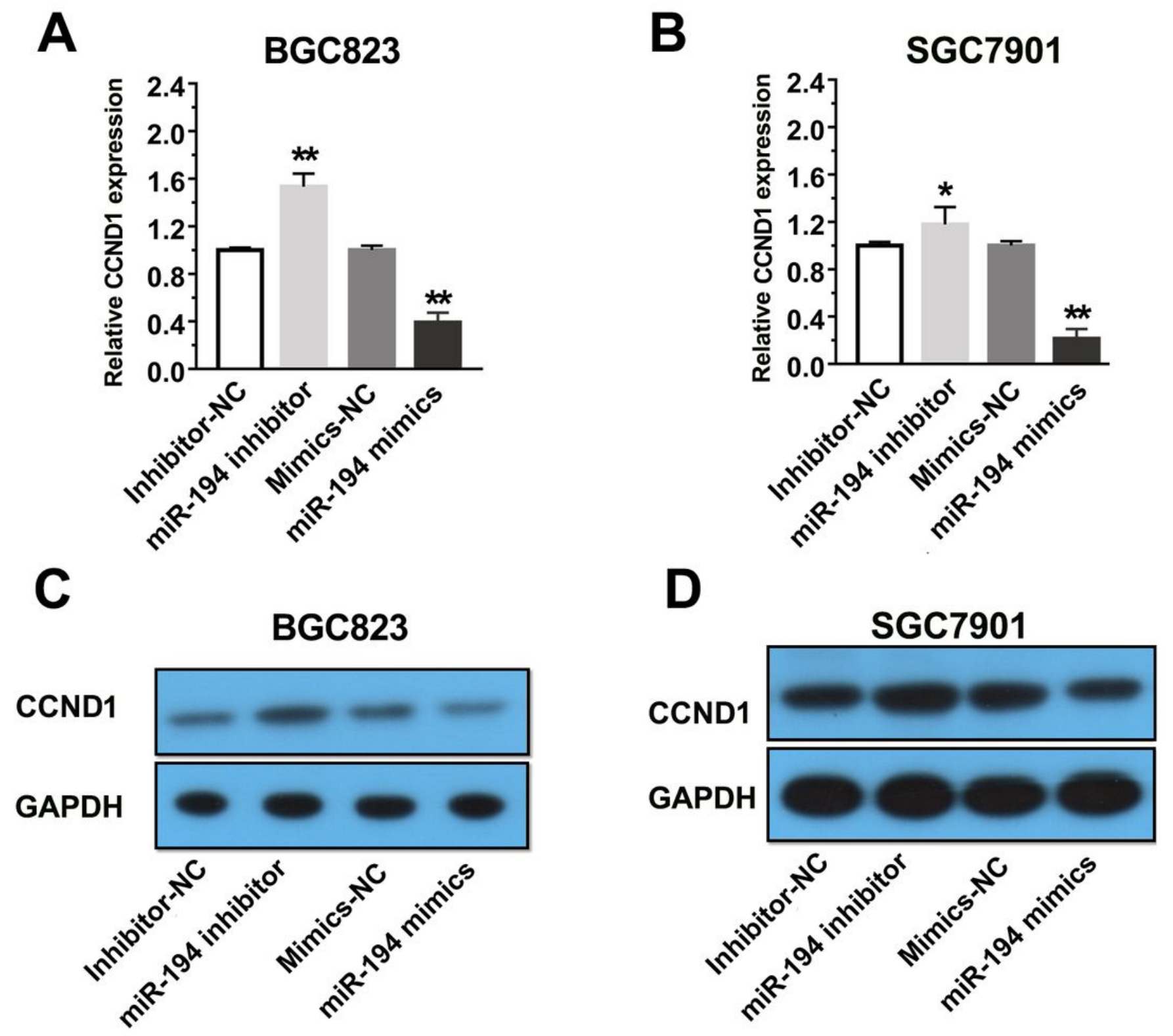

Figure 4

CCND1 was negatively regulated by miR-194 in GC. (A) Determination of CCND1 expression level in the BGC823 cells that transfected with miR-194 mimics and inhibitors by qRT-PCR assay. (B) Determination of CCND1 expression level in the SGC7901 cells that transfected with miR-194 mimics and inhibitors by qRT-PCR assay. (C) Determination of CCND1 protein level in the BGC823 cells that transfected with miR- 
194 mimics and inhibitors by western blot assay. (D) Determination of CCND1 protein level in the BGC823 cells that transfected with miR-194 mimics and inhibitors by western blot assay. $\left({ }^{*}, \mathrm{P}<0.05\right.$; $\left.* *, \mathrm{P}<0.01\right)$

A

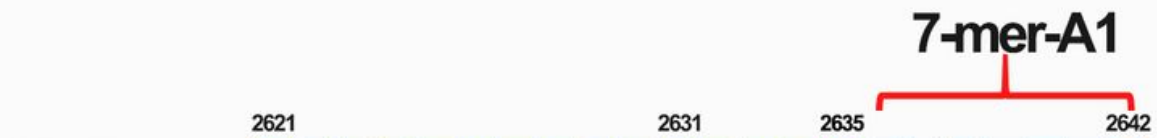

CCND1 5,-ACCT T C T G C C T T T GA T G T TA CA-3'

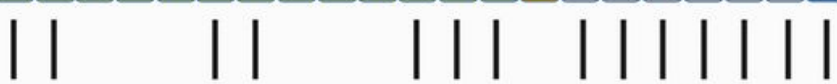

miR-194 3'-A G G T G T A C C T C A A C G A CA A T G T -5'

$\triangle \mathbf{G}$ ( folding energy)

-9 (-Kcal/mol)

B

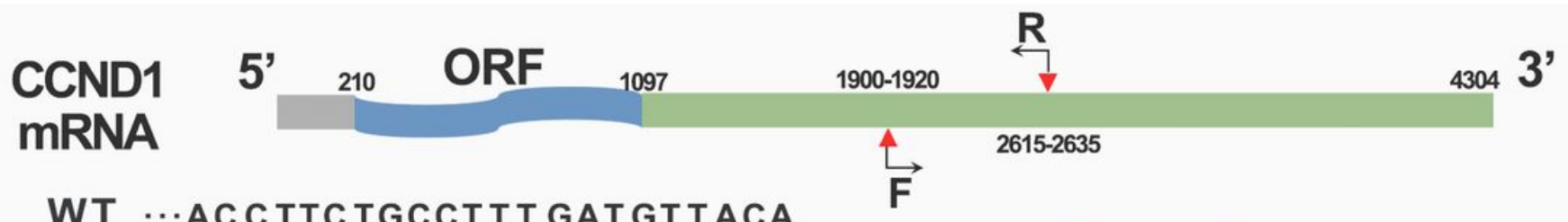

WT $\cdots$ ACCTTCTGCCTTTGATGTTACA

| | | | | |

miR-194 …AGGTGTACC T CAACGACAAT GT

| | |

$R w T=R+T G T T A C A$

Mut $\cdots$ AC

$$
R_{\text {Mut }}=R+\text { AAAAACA }
$$

C

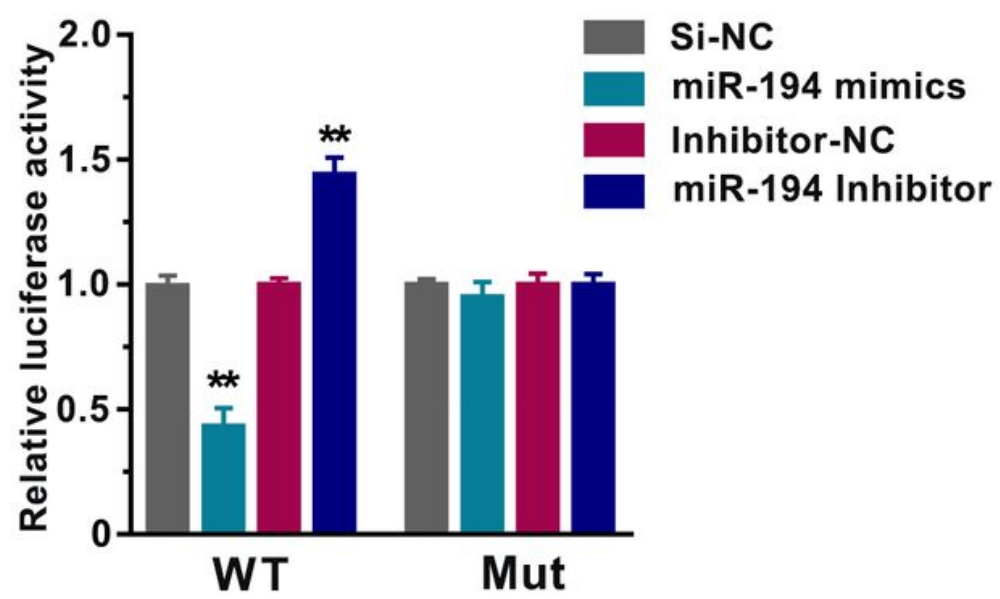

Figure 5

The CCND1 gene was a target gene of miR-194 in GC. (A) The binding mode and folding energy between miR-194 and CCND1 was predicted by MiRcode and RNA22 web-based tool. (B) The schematic diagram of construction of wildtype luciferase reporter vector and the mutant luciferase reporter vector. (C) The BGC823 cells were co-transfected with miR-194 mimics (or inhibitors) and wildtype (or mutant) luciferase reporter vector. After $48 \mathrm{~h}$ of incubation, luciferase activity was measured. (**, $\mathrm{P}<0.01$ ) 

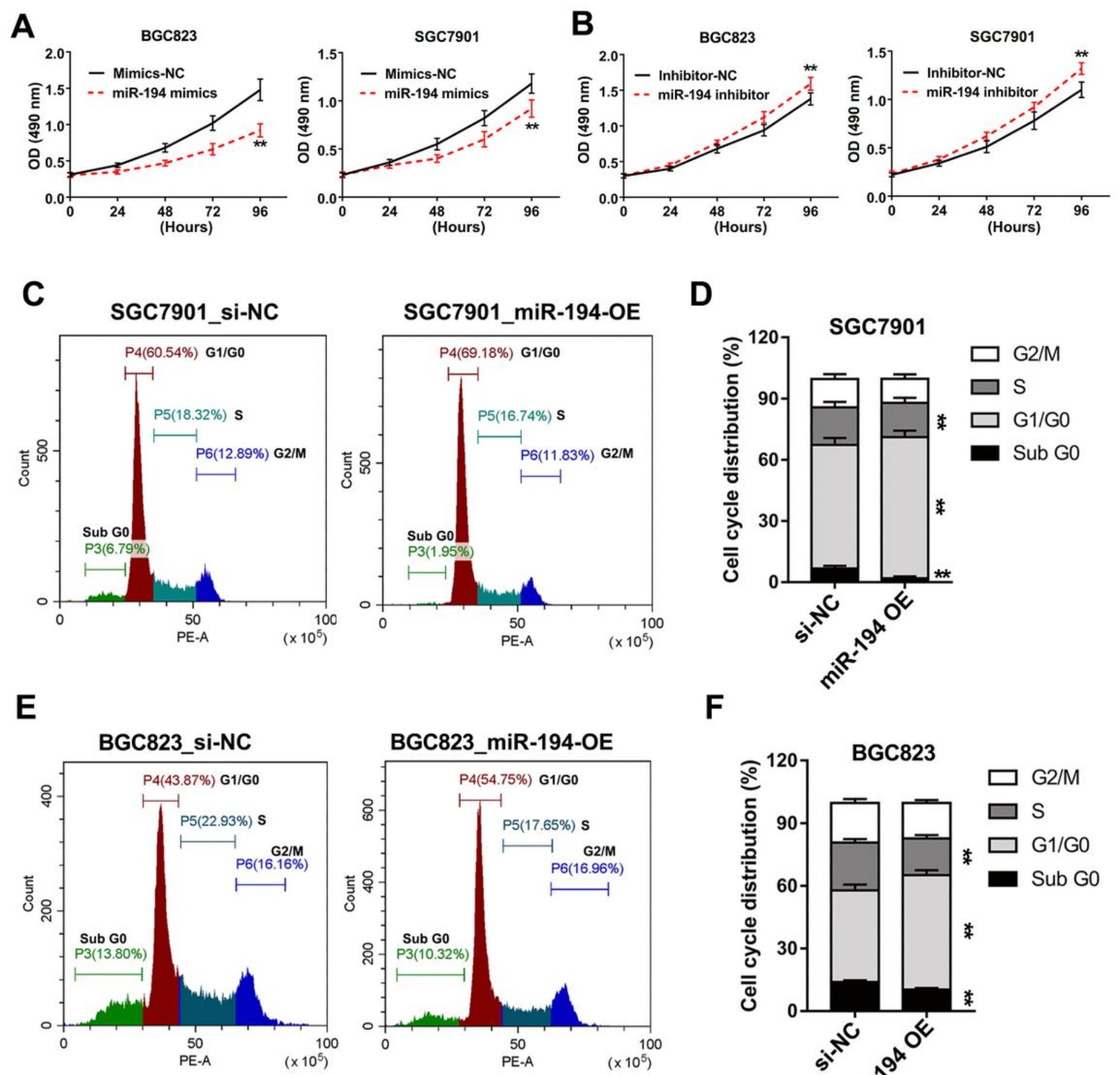

$\mathbf{F}$

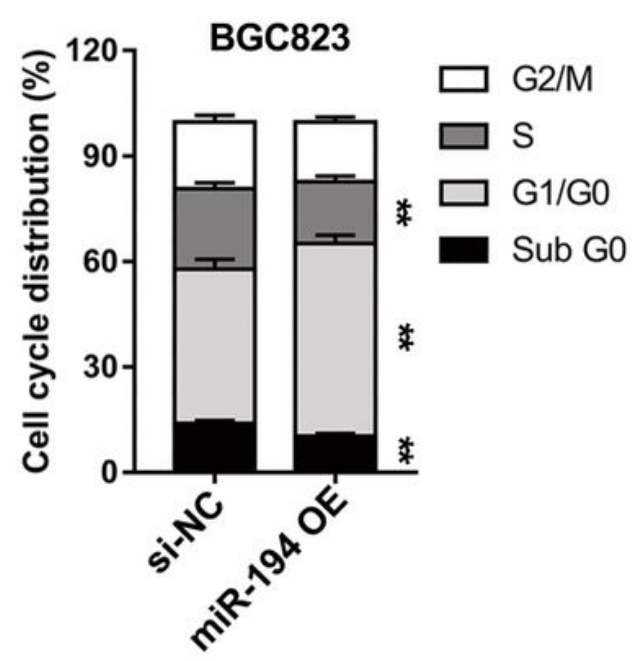

Figure 6

The GC cell growth and cell cycles were impaired by miR-194. (A) The CCK-8 proliferation assay in SGC7901 and BGC823 cells that transfected with miR-194 mimics. (B) The CCK-8 proliferation assay in SGC7901 and BGC823 cells that transfected with miR-194 inhibitors. (C) Flow cytometric analysis was performed in SGC7901 cells that transfected with miR-194 mimics. (D) The data of cell cycle analysis were descripted as percentage distribution of cells in subG0, G0/G1, S and G2/M phases of the cell cycle. (E) Flow cytometric analysis was performed in SGC7901 cells that transfected with miR-194 mimics. (F) 
The data of cell cycle analysis were descripted as percentage distribution of cells in subG0, G0/G1, S and $\mathrm{G} 2 / \mathrm{M}$ phases of the cell cycle. All flow cytometry assays were reproducible in three independent experiments. $(* *, \mathrm{P}<0.01)$

\section{Supplementary Files}

This is a list of supplementary files associated with this preprint. Click to download.

- TableS1.docx

- FigS1.jpg 